\title{
Cell-cell and Cell-noise Interactions of Bacterial Cells in a Shallow Circular Pool and Transitions of Collective Motions
}

\author{
Ryojiro Honda, Sora Umeda, and Jun-ichi Wakita \\ Department of Physics, Chuo University, Bunkyo, Tokyo 112-8551, Japan
}

\begin{abstract}
We have experimentally investigated the transitions of collective motions of bacterial cells in a shallow circular pool using the bacterial species Bacillus subtilis. In our previous paper, we reported that the collective motions were classified into six phases on a phase diagram with two parameters, namely, the reduced cell length $\lambda$ and the cell density $\rho$. In this study, we focused on the sharp transitions at $\lambda \cong 0.1\left(\equiv \lambda_{\mathrm{C} 1}\right)$ with low values of $\rho$ between the random motion phase and the one-way rotational motion phase. By introducing the order parameter $Q$, which measures the aligned cell motion along the circumferential direction of a pool, the transitions at $\lambda=\lambda_{\mathrm{C} 1}$ were clearly characterized. On the basis of the detailed observations of single-cell trajectories in a pool, we verified that the effect of cell-noise interactions was widely distributed. We conclude that, even in such random environment, the sharp transitions of collective motions were caused by the cell-cell interactions at $\lambda=\lambda_{\mathrm{C} 1}$.
\end{abstract}

\section{Introduction}

Various types of ordered behavior including the construction of vortexlike structures were observed in flocks of birds, schools of fish, marches of social insects, and migrations of bacteria!19) It was also reported that elongating bacteria produce complicated structures in folding and filling processes on an agar surface by cell multiplications. 10 [11)

Migrating bacteria in a growing colony show a variety of collective behavior depending on environmental conditions, although a bacterial colony is one of the simplest biological systems. 12 15) Vicsek et al. proposed a statistical mechanics model called the self-propelled particle (SPP) model to demonstrate such collective motions. $\frac{16}{20}$ Their model shows the dynamical phase transition such that the ordered motions with aligned directions suddenly emerge from chaotic motions in the self-propelled particle system at a critical noise amplitude or at a critical particle density. The origin of the transition is considered to be the competition between the effect that each particle tends to be aligned in the averaged direction of its neighboring particles and the individual fluctuations of moving directions due to noises. The hydrodynamic motions of cells including the creation and annihilation of vortices have also been universally observed in growing colonies of several bacterial species. ${ }^{[6]}$ ) Wioland et al. reported that cells in a highly concentrated bacterial 
droplet form a single stable vortex that can rotate in both clockwise and counterclockwise directions in the droplet.21) By comparing their experimental results with a model, they argued that the global confinement condition and the curvature of droplet boundaries play important roles in realizing such a steady state having a single vortex in the system. Similar self-organized structures involving vortices and clusters have also been reported in other confinement systems.22 27) In an experimental study, however, it is generally difficult to verify which is essential for the emergence of collective motions among cell-cell interactions, cell-boundary interactions, and individual fluctuations due to noises.

In our previous study, we experimentally investigated the collective motions of bacterial cells in a shallow circular pool using the bacterial species Bacillus (B.) subtilis. ${ }^{28}$ ) The pools were made on the surface of an agar plate, in which the collective motions of rod-shaped bacterial cells were observed. We introduced two relevant parameters, namely, the reduced cell length $\lambda$, which was defined as the ratio of the averaged cell length to the pool diameter, and the average cell density $\rho$. As shown by the phase diagram (Fig. 1), the observed collective motions have been classified into six dynamical phases: (a) random motion, (b) turbulent motion, (c) one-way rotational motion, (d) two-way rotational motion, (e) random oscillatory motion, and (f) ordered oscillatory motion. In the random motion phase, bacterial cells move independently and randomly in a pool. In the turbulent motion phase, bacterial cells form groups showing hydrodynamic motions with the creation and annihilation of vortices. In the one-way rotational motion phase, bacterial cells move counterclockwise along the brim of a pool keeping their axes parallel with the brim. This suggests that the brim of a pool acts as a nearly slip boundary for a bacterial cell. Note that in Ref. 21, however, the interface of a droplet acts as a nearly no-slip boundary. In the two-way rotational motion phase, bacterial cells move counterclockwise in the outer region of a pool and clockwise in the inner region of a pool. In the random oscillatory motion phase, bacterial cells move forward and backward repeatedly in their axial directions. In this phase, the axial oscillatory motions of bacterial cells seem to be individually random and uncorrelated. In the ordered oscillatory motion phase, on the other hand, if we observe the system for a sufficiently long time, intermittent ordering of the axial directions of bacterial cells is observed. As indicated in Fig. 1 by two dashed lines perpendicular to the $\lambda$ axis, there are two critical values of $\lambda, \lambda_{\mathrm{C} 1} \cong 0.1$ and $\lambda_{\mathrm{C} 2} \cong 0.2$. By crossing these two lines in the phase diagram, marked changes in collective motions occur.

In this study, we focused on the transitions at $\lambda=\lambda_{\mathrm{C} 1}$ at the low values of $\rho$ between the random motion phase and the one-way rotational motion phase. The transitions at $\lambda=\lambda_{\mathrm{C} 1}$ were characterized by introducing the order parameter $Q$, which measures the aligned cell motion in the circumferential direction of a pool. If the reduced cell length $\lambda$ increases with a constant cell density $\rho$, then more collisions between cells occur and the alignment effect of neighboring cells is enhanced. In this sense, $\lambda$ is a parameter representing the coupling strength of cell-cell interactions. 
To study cell-cell interactions, we have examined the systems in which only a single bacterial cell is moving in a pool. As a matter of course, no cell-cell interactions exist in such single cell systems. The parameter $\tilde{Q}$ for the one-way rotational motion in the single cell systems, which was defined as the time-averaged velocity component parallel to the brim of a pool, did not show any systematic dependence on $\lambda$. We found instead that $\tilde{Q}$ approximately linearly depended on the time-average speed $v_{\text {ave }}$ of a bacterial cell. We verified that $v_{\text {ave }}$ was determined by the frequency of the change in the moving direction in each trajectory of a cell. The change in the moving direction of a cell will be attributed to the cell-noise interactions between a cell and irregularities on the surface of a shallow pool, which cannot be controlled in our experimental setting.

Combining the experimental results of the original systems with a large number of bacterial cells and the single cell systems, we conclude that the transitions at $\lambda=\lambda_{\mathrm{C} 1}$ from the random motion phase to the one-way rotational motion phase were caused by the cell-cell interactions in the systems involving uncontrolled cell-noise interactions.

All through the experiments, we used the wild-type strain OG-01 of B. subtilis. $\frac{12}{12} B$. subtilis cells are rod-shaped with peritrichous flagella and swim straightforwardly in water by bundling and rotating their flagella. When a small number of cells are inoculated on the surface of semisolid agar plates, they form a colony by cell motility and multiplication. Growing colonies present five different patterns depending on two environmental parameters $C_{\mathrm{a}}$ (agar concentration) and $C_{\mathrm{n}}$ (nutrient concentration) ${ }^{12}$ ) In particular, when $C_{\mathrm{a}}$ is intermediate $\left(7 \mathrm{~g} / \mathrm{L}<C_{\mathrm{a}}<8.5 \mathrm{~g} / \mathrm{L}\right)$ and $C_{\mathrm{n}}$ is high $\left(C_{\mathrm{n}}>10 \mathrm{~g} / \mathrm{L}\right)$, the interface of a growing colony repeatedly advances (in the migration phase) and rests (in the consolidation phase), and finally yields a concentric-ring pattern. The bacterial cells at the growing front of a concentric-ring pattern have been observed to repeat elongation and contraction, synchronizing with the periodic colony growth.12 (29)

Shallow circular pools are made on the surface of an agar plate by scattering glass beads on the growing front of a concentric-ring pattern and then removing the glass beads. ${ }^{280}$ Bacterial cells trapped in a pool show a variety of collective motions. We can control $\lambda$ by changing the timing of making pools on the growing front, since the cell length of bacteria differs at different times in the growing front in the concentric-ring pattern formation. Thanks to the local fluctuation of the cell density at the growing front, a variety of pools with different cell densities $\rho$ can be made.

This paper is organized as follows. In Sect. 2, we explain the experimental procedures. Experimental results are given in Sect. 3. In Sect. 4, we discuss our experimental results and future problems.

\section{Experimental Procedure}

First, we prepared semisolid agar plates by following the procedures explained below. $5 \mathrm{~g}$ of sodium chloride $(\mathrm{NaCl}), 5 \mathrm{~g}$ of dipotassium hydrogen phosphate $\left(\mathrm{K}_{2} \mathrm{HPO}_{4}\right)$, and $30 \mathrm{~g}$ of Bacto-Peptone (Becton, Dickinson and Co.) as nutrient were dissolved in $1 \mathrm{~L}$ 


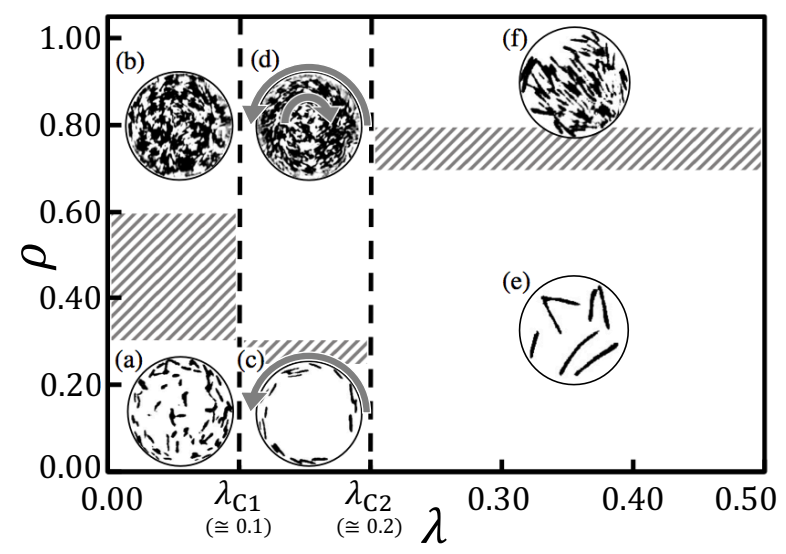

Fig. 1. Phase diagram of collective motions of bacterial cells in a shallow circular pool with two parameters $\lambda$ and $\rho$ : (a) random motion phase, (b) turbulent motion phase, (c) one-way rotational motion phase, (d) two-way rotational motion phase, (e) random oscillatory motion phase, and (f) ordered oscillatory motion phase. The rotational directions in (c) and (d) are shown by the arrows. The two vertical dashed lines show the critical values $\lambda_{\mathrm{C} 1} \cong 0.1$ and $\lambda_{\mathrm{C} 2} \cong 0.2$, at which the collective motions are changed considerably.

of distilled water. The environmental parameter $C_{\mathrm{n}}$ was set to $30 \mathrm{~g} / \mathrm{L}$ by adjusting the concentration of Bacto-Peptone. Then, the solution was adjusted to $\mathrm{pH} 7.1$ by adding $6 \mathrm{~N}$ hydrochloric acid $(\mathrm{HCl})$. Furthermore, the solution was mixed with $8.3 \mathrm{~g}$ of Bacto-Agar (Becton, Dickinson and Co.), which determines the softness of the semisolid agar plates. The environmental parameter $C_{\mathrm{a}}$ was set to $8.3 \mathrm{~g} / \mathrm{L}$ by adjusting the concentration of Bacto-Agar. The environmental condition given by these $C_{\mathrm{a}}$ and $C_{\mathrm{n}}$ values generates a typical concentric-ring pattern of $B$. subtilis colonies. The solution was autoclaved at $121{ }^{\circ} \mathrm{C}$ for $15 \mathrm{~min}$, and $20 \mathrm{ml}$ of the solution was poured into each sterilized plastic petri dish of $88 \mathrm{~mm}$ inner diameter. The thickness of the semisolid agar plates was about 3.2 $\mathrm{mm}$. After solidification at room temperature for $60 \mathrm{~min}$, the semisolid agar plates were dried at $50{ }^{\circ} \mathrm{C}$ for $90 \mathrm{~min}$.

Next, we prepared a bacterial suspension with an optical density of 0.5 at a wavelength of $600 \mathrm{~nm}$. We inoculated $3 \mu \mathrm{l}$ of the bacterial suspension on the surface of each semisolid agar plate. The optical density of 0.5 corresponds to a bacterial density of about $10^{4}$ cells per $\mu l$. The semisolid agar plates were left at room temperature for about 60 min to dry the bacterial suspension droplet.

Then, we incubated the semisolid agar plates in a humidified box at $35{ }^{\circ} \mathrm{C}$ and $90 \%$ RH. Bacterial cells at the inoculation spot grew and multiplied by cell division without migration during the lag phase period of about $7 \mathrm{~h}$. After the lag phase, the first migration started and two-dimensional colony expansion was observed. About 2 h later, they stopped migrating and entered the first consolidation phase. They did not move but underwent cell division actively for about $5 \mathrm{~h}$. Afterwards, they exhibited the migra- 
tion phase and the consolidation phase alternately. Thus, we obtained a concentric-ring pattern of the bacterial colonies.

After that, we scattered glass beads of $50 \pm 2 \mu \mathrm{m}$ diameter (Unitika, SPM-50) in the vicinity of the growing front of a concentric-ring pattern at the start of the third migration phase or the third consolidation phase of colony growth. After we removed the beads from the agar surface using adhesive tape, circular pools were made on the surface. Bacterial cells under a bead were trapped and were moving in a pool ${ }^{28}$ ) The pool depth was about $1 \mu \mathrm{m}$, which was much smaller than the pool diameter. The thickness of each bacterial cell was about $0.5 \mathrm{\mu m}$, which was approximately the same scale of the pool depth. As a result, two-dimensional motions of bacterial cells were realized in the pools. During an observation period, which was set to be $10 \mathrm{~min}$, corresponding to the half of a cell division cycle, the cell length was almost constant. We have confirmed that there was no inflow of bacterial cells into a pool and no outflow of bacterial cells from a pool. Therefore, the cell density $\rho$ was almost constant and the collective motions of bacterial cells were stationary during an observation period. Furthermore, water was always supplied to a pool from the agar surface, so that we were able to observe the motions of bacterial cells for relatively long time periods, which were typically more than $10 \mathrm{~min}$.

We observed and video-recorded the collective motions of bacterial cells at $30 \mathrm{~Hz}$ with a high-speed microscope (Keyence, VW-9000) linked to an optical microscope (Nikon, DIAPHOT-TMD). We defined the area of a circular pool as the area covered by the trajectories traced by bacterial cells in the time duration of $10 \mathrm{~min}$. The pool diameter was calculated from the area of a pool. In the above analysis, we used an image and motion analysis library (OpenCV). On the other hand, we measured cell lengths in a snapshot captured from the video by hand. The cell density in each pool was given by dividing the total area of bacterial cells in a pool in a snapshot by the area of a pool. To acquire the velocity field of bacterial motion in the random motion phase and the oneway rotational motion phase, we used particle image velocimetry (PIV) software (Library, Flow-PIV).

\section{Experimental Results}

\subsection{Transition between random motion phase and one-way rotational motion phase}

\subsubsection{Introducing order parameter $Q$}

To clarify the differences between the cell motions in the random motion phase and in the one-way rotational motion phase, we focused on the circumferential direction component of the cell velocity in a pool as explained in the following.

We performed time-series measurements at intervals of $1 / 30 \mathrm{~s}$ of the bacterial velocity fields in the range of $0.05<\lambda<0.20$. One observation period is $60 \mathrm{~s}$, so that each timeseries measurement consists of 1,800 discrete velocity fields. The typical velocity fields of cell motions in the random motion phase and in the one-way rotational motion phase are shown in the snapshots in Figs. 2(a) and 2(b), respectively. 
We put the origin of two-dimensional coordinates at the center of a circular pool and introduce the polar radius $r$ and the polar angle $\theta$. To analyze the velocity fields, we consider a lattice of a square mesh of $1.88 \mu \mathrm{m}$ spacing on a circular pool and use the polar coordinates $(r, \theta)$ to specify the lattice points. At each lattice point $(r, \theta), \hat{\boldsymbol{S}}(r, \theta)$ is defined as a unit tangent vector in the circumferential direction of the pool, where the positive direction is assumed to be in the counterclockwise direction.

At each time $t$, the velocity vectors $\boldsymbol{V}(t ; r, \theta)$ were measured and their normalized vectors $\hat{\boldsymbol{V}}(t ; r, \theta)$ were obtained by $\boldsymbol{V}(t ; r, \theta) /|\boldsymbol{V}(t ; r, \theta)|$ at all lattice points $(r, \theta)$. Then we defined the local order parameter $q(t ; r, \theta)$ by the inner product

$$
q(t ; r, \theta)=\hat{\boldsymbol{V}}(t ; r, \theta) \cdot \hat{\boldsymbol{S}}(r, \theta)
$$

at each time $t$ and at each lattice point $(r, \theta)$. Then for each $r$, we averaged $q(t ; r, \theta)$ over the observation time-period $T=60 \mathrm{~s}$ (by summing over 1,800 discrete velocity fields at different times) and averaged it over polar angles $\theta \in[0,2 \pi)$. That is, we obtained a discretized approximation on the lattice for the quantity

$$
q(r)=\frac{1}{T} \int_{0}^{T} d t \frac{1}{2 \pi} \int_{0}^{2 \pi} d \theta q(t ; r, \theta) .
$$

Since the radius $a$ of a pool was distributed, 28 we introduced the normalized radius coordinate as $R=r / a$. The $R$-dependent order parameter was then defined as

$$
Q(R)=q(a R)
$$

By the definition mentioned above, if all cells are undergoing counterclockwise one-way rotational motion at any place in a pool, we have $Q(R)=1$ for each $R \in(0,1)$, while if cell motions are completely random, $Q(R)=0$ for each $R \in(0,1)$. Hence, $Q(R)$ will act as the order parameter for the one-way rotational motion.

Figure 3(a) shows the profiles of $Q(R)$ for $\lambda=0.07<\lambda_{\mathrm{C} 1}$ and $\lambda=0.17>\lambda_{\mathrm{C} 1}$, where $\lambda_{\mathrm{C} 1} \cong 0.1$. The profiles have a maximum value at $R \simeq 0.8$, while $Q(R) \simeq 0$ for $R<0.5$. From now on, we focus on the maximum value, and define the order parameter $Q$ for the one-way rotational motion as

$$
Q=\max _{R} Q(R)
$$

\subsubsection{Behavior of order parameter $Q$}

Figure 3 (b) shows the $\lambda$-dependence of the order parameter $Q$ in the vicinity of $\lambda_{\mathrm{C} 1}$. There, we plotted $Q$ only for the range of $0.11 \leq \rho \leq 0.30$ for the following reasons. The upper limit $\rho \simeq 0.30$ is the threshold value between the one-way rotational motion phase and the two-way rotational motion phase as shown in Fig. 1. In the one-way rotational motion phase, bacterial cells were often observed near the brim of the pool, so that the local cell density near the brim was effectively higher than the density $\rho$ averaged over 
the whole pool. When $\rho \geq 0.11$, bacterial cells filled the outer region of the pool and the one-way rotational motion became stable. As shown in Fig. 3(b), $Q$ remains at small values $\simeq 0.3$ for $\lambda \leq \lambda_{\mathrm{C} 1} \cong 0.1$, while it is evident that $Q$ increases with $\lambda$ for $\lambda \geq \lambda_{\mathrm{C} 1}$. The quantity $Q=Q(\lambda)$ indeed acts as the order parameter for the one-way rotational motion. (Strictly speaking, the order parameter for one-way rotational motion should be zero for random motion. A possible improvement of the definition of the order parameter is proposed in Sect. 4.)

(a)

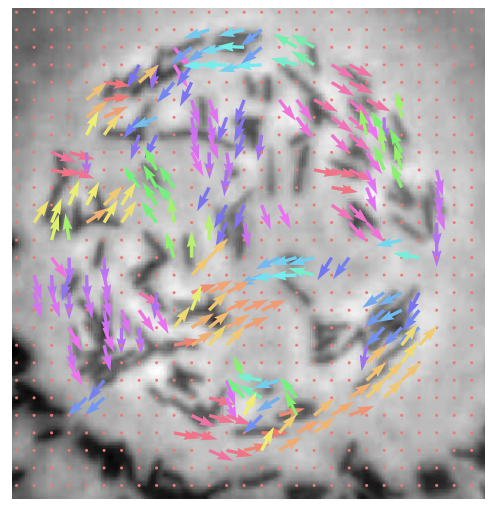

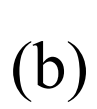

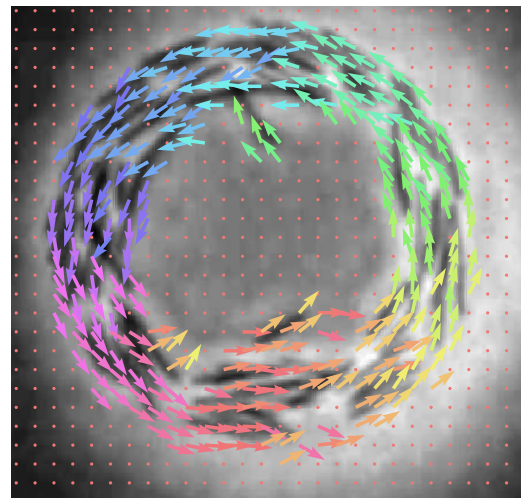

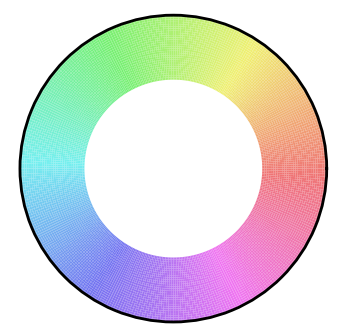

Fig. 2. (Color online) Typical velocity fields for the random motion phase at $\lambda=0.07$ in (a) and for the one-way rotational motion phase at $\lambda=0.17$ in (b). Velocity fields overlaid on the snapshots of bacterial cells, in which the orientations of velocity vectors are indicated by arrows with colors assigned as shown by the color ring.

\subsection{Effects of cell-cell interactions and fluctuation of bacterial motions}

\subsubsection{Characterization of bacterial motions in single cell systems}

To examine the effects of cell-cell interactions on the transitions between the random motion phase and the one-way rotational motion phase, we have examined single cell systems with only one bacterial cell moving in each pool. In the original systems with many bacterial cells in each pool, the cell-cell interactions will be effective. They may cause the tendency of the bacterial motions to make the individual direction of motion be aligned with the averaged moving direction of the neighboring cells, and also the repulsive effects between bacterial cells. As a matter of course, no cell-cell interactions exist in the single cell systems.

First, we tracked a bacterial cell in each single cell system at intervals of $1 / 30 \mathrm{~s}$ for the time duration of $600 \mathrm{~s}$. Again, we used the two-dimensional polar coordinate system with the origin set at the center of the pool. For each trajectory of the geometric center of a cell, we obtained a time series of 18,000 polar coordinates $(r(t), \theta(t))$. Figure 4(a) shows a typical trajectory for $1 \mathrm{~s}$.

Then, we evaluated the time-series velocity vectors $\boldsymbol{v}(r(t), \theta(t))$ from the differences in successive polar coordinates $(r(t), \theta(t))$. We calculated the inner products of the 

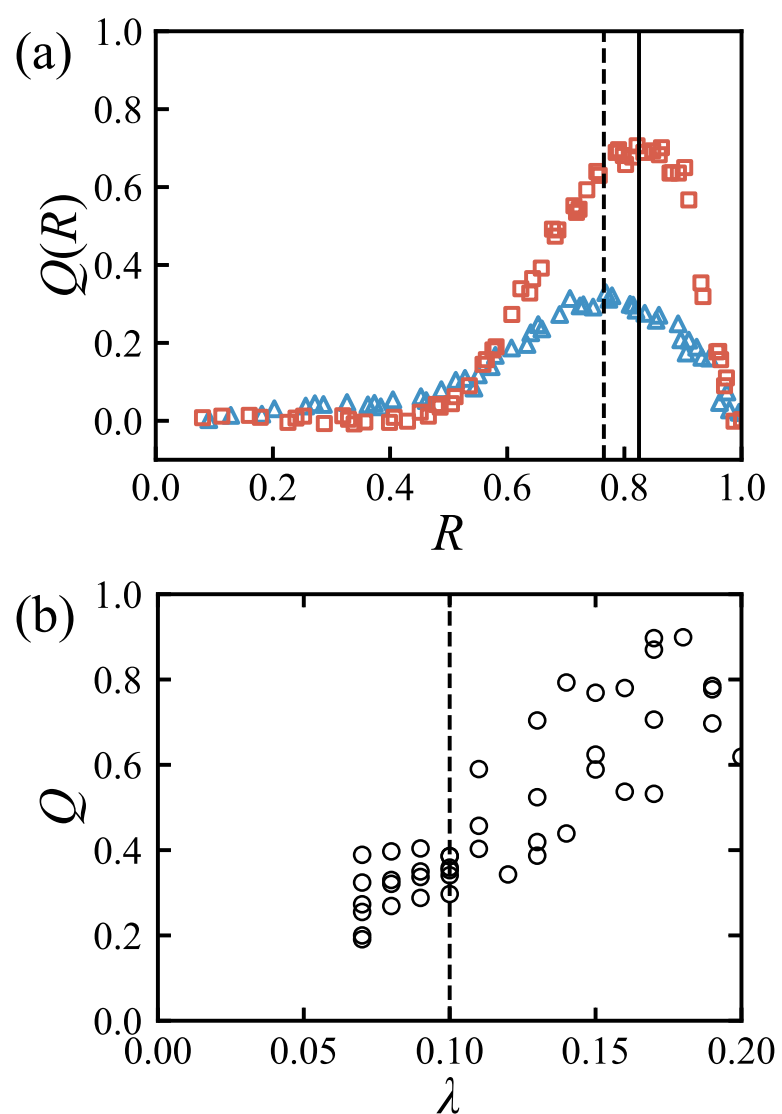

Fig. 3. (Color online) Measurement of the order parameter $Q$. (a) Profiles of $Q(R)$ for $\lambda=0.07<\lambda_{\mathrm{C} 1}$ (blue triangles) and for $\lambda=0.17>\lambda_{\mathrm{C} 1}$ (red squares), where $\lambda_{\mathrm{C} 1} \cong 0.1$. Both profiles have maximum values at $R \simeq 0.8$, while they have $Q(R) \simeq 0$ for $R<0.5$. (b) Values of $Q$ plotted against $\lambda$ for $0.11 \leq \rho \leq 0.30$. For $\lambda \leq \lambda_{\mathrm{C} 1} \cong 0.10, Q$ remains at small values $\simeq 0.3$, while $Q$ evidently increases with $\lambda$ for $\lambda \geq \lambda_{\mathrm{C} 1}$. 
normalized velocity vectors $\hat{\boldsymbol{v}}(r(t), \theta(t))$ defined as $\boldsymbol{v}(r(t), \theta(t)) /|\boldsymbol{v}(r(t), \theta(t))|$ and the unit tangent vectors $\hat{\boldsymbol{s}}(r(t), \theta(t))$ defined as the unit vectors in the circumferential direction of a pool at the polar coordinates $(r(t), \theta(t))$. The positive direction of $\hat{\boldsymbol{s}}(r(t), \theta(t))$ is assumed to be in the counterclockwise direction. We examined the behavior of the time average of the inner products defined as

$$
\tilde{Q}(T)=\frac{1}{T} \sum_{t=0}^{T} \hat{\boldsymbol{v}}(r(t), \theta(t)) \cdot \hat{\boldsymbol{s}}(r(t), \theta(t))
$$

by changing the averaging time duration $T$. Figure $4(\mathrm{~b})$ shows $\tilde{Q}(T)$ as a function of $T$. This asymptotically approached the value for $T=600 \mathrm{~s}$, which is shown by the dotted line in Fig. 4(b). By this consideration, we defined the parameter $\tilde{Q}$ for the one-way rotational motion in the single cell systems by $\tilde{Q}(600) ; \tilde{Q}=\tilde{Q}(600)$. If a bacterial cell moves counterclockwise in the circumferential direction of a pool, we have $\tilde{Q}=1$, whereas if a bacterial cell moves completely randomly, $\tilde{Q}=0$.

\subsubsection{Behavior of parameter $\tilde{Q}$}

Figure 5(a) shows plots of the parameter $\tilde{Q}$ against $\lambda$ in the range of $0<\lambda<0.20$. Note that $\tilde{Q}$ is always positive, which implies that bacterial cells tend to move counterclockwise in the circumferential direction of their pool. In the microscopic observation, when bacterial cells moved counterclockwise along the brim of a pool, the axes of the cells were parallel to the brim. On the other hand, when bacterial cells sometimes moved clockwise, the axes were not parallel to the brim and kept a certain angle against the brim. Bacterial cells seemed unable to swim clockwise smoothly along the brim. The difference in the axial directions to the brim between counterclockwise motion and clockwise motion is due to some biological characteristics of the present species of bacterial cells, hence we regard the interactions between a cell and the brim as the cell-boundary interactions. The values of $\tilde{Q}$ were widely distributed between 0 and 1 , and seemed to have no systematic dependence on $\lambda$. The critical value $\lambda_{\mathrm{C} 1}$ found in Fig. 3(b) for the collective motions becomes meaningless in the single cell systems.

Then, we studied the average of bacterial cell speeds $v(=|\boldsymbol{v}(r(t), \theta(t))|)$ over the time duration of $600 \mathrm{~s}$, which was denoted as $v_{\text {ave }}$. Figure 5 (b) shows the plots of the parameter $\tilde{Q}$ against the time-average speed $v_{\text {ave }}$. $\tilde{Q}$ seemed to increase in proportion to $v_{\text {ave }}$. The distributions of cell speed $v$ and the trajectories of a bacterial cell for the colored plots in Fig. [5) (b) are shown in Fig. [6 in the next section.

\subsubsection{Distributions of cell speed $v$}

In order to know what determines the time-average speed $v_{\text {ave }}$ of a bacterial cell in the single cell systems, we compared the distributions of cell speed $v$ for different values of $v_{\text {ave }}$. Figure 6(a) shows the histograms of the cell speed $v$ for different values of $v_{\text {ave }}$. Each histogram was made from 18,000 successive cell speeds $v$. The bin range was set from 0 to $100 \mathrm{\mu m} / \mathrm{s}$ with a bin width of $1 \mu \mathrm{m} / \mathrm{s}$. The total area of the histograms was normalized 
to one, so that they give probability densities. The three vertical lines indicate the values of $v_{\text {ave }}$ for each histogram, which are $20.5 \mu \mathrm{m} / \mathrm{s}$ (dotted line), $35.2 \mu \mathrm{m} / \mathrm{s}$ (dashed line), and $51.1 \mathrm{\mu m} / \mathrm{s}$ (solid line) from the left. These values of $v_{\text {ave }}$ were also shown by the colored symbols in Fig. [5)(b). The probability densities of $v$ had two peaks. One of them was at $v \cong 0 \mu \mathrm{m} / \mathrm{s}$ and the other one was at $v>30 \mu \mathrm{m} / \mathrm{s}$. When the probability density at $v \cong 0 \mu \mathrm{m} / \mathrm{s}$ was low, that at $v>30 \mu \mathrm{m} / \mathrm{s}$ was high and then $v_{\text {ave }}$ was large. When the probability density at $v \cong 0 \mu \mathrm{m} / \mathrm{s}$ was high, that at $v>30 \mu \mathrm{m} / \mathrm{s}$ was low and then $v_{\text {ave }}$ was small. These tendencies suggest that $v_{\text {ave }}$ strongly depends on the relationship between the two peaks. The trajectories of a bacterial cell for the different values of $v_{\text {ave }}$ in Fig. 6(a) are shown for $60 \mathrm{~s}$ in Fig. 6(b). Namely, the left, middle, and right pictures in Fig. 6(b) correspond to the distributions of $v$ for $v_{\text {ave }}=20.5,35.2$, and $51.1 \mu \mathrm{m} / \mathrm{s}$ in Fig. 6(a), respectively. As $v_{\text {ave }}$ increased, the trajectories seemed to become less complicated, that is, the fluctuation of the bacterial motion decreased.

Here, we examined the dependence of $v_{\text {ave }}$ on $\lambda$. Figure 6 (c) shows that the values of $v_{\text {ave }}$ were widely distributed between 10 and $60 \mu \mathrm{m} / \mathrm{s}$, and did not seem to have any systematic dependence on $\lambda$. This result is consistent with the results shown in Figs. 5(a) and 5 (b) and with the possibility that $v_{\text {ave }}$ depends on the relationship between the two peaks.

\section{Discussion and Future Problems}

In Sect. 3.1.2, we studied the transitions of the collective motions of bacterial cells in a shallow circular pool between the random motion phase and the one-way rotational motion phase when the reduced cell length $\lambda$ was changed in the vicinity of $\lambda_{\mathrm{C} 1}$. From the dependence of $Q$ on $\lambda$ shown in Fig. 3(b), a change in the behavior of $Q$ was recognized at $\lambda=\lambda_{\mathrm{C} 1}$. In the microscopic observation for $\lambda \geq \lambda_{\mathrm{C} 1}$ (in the one-way rotational motion phase), we found that the bacterial cells were localized in the outer region of their pool and were moving counterclockwise along the brim of the pool. (The outer region is defined as the range of $0.6 \leq R \leq 1.0$ from the profile of $Q(R)$ in Fig. $3(\mathrm{a})$.) As shown in Fig. 3(a), the value of $R$ at which $Q(R)$ attained its maximum was about 0.8. Therefore, the order parameter $Q$ defined by $\max _{R} Q(R)$ indicates a typical value of $Q(R)$ for bacterial cell motions. On the other hand, from the microscopic observation, we found that also in the systems with $\lambda \leq \lambda_{\mathrm{C} 1}$ (in the random motion phase), the bacterial cells in the outer region of the pool tended to move counterclockwise along the brim of the pool. This tendency is considered to be due to the cell-boundary interactions. As a result, the values of $Q$ were in the range of $0.2 \leq Q \leq 0.4$ even for $\lambda \leq \lambda_{\mathrm{C} 1}$ as shown in Fig. 3(a). We considered, however, that the typical behavior of bacterial cell motions for $\lambda \leq \lambda_{\mathrm{C} 1}$ should be measured in the inner region of a pool apart from the cell-boundary interactions. Thus, here we redefine the order parameter $Q$ for $\lambda \leq \lambda_{\mathrm{C} 1}$ as the value of $Q(R)$ at $R=0.3$ (the middle position of the inner region). Figure 7 shows the dependence of $Q$ on $\lambda$, in which the values of $Q$ became almost zero for $\lambda \leq \lambda_{\mathrm{C} 1}$. 
(a)
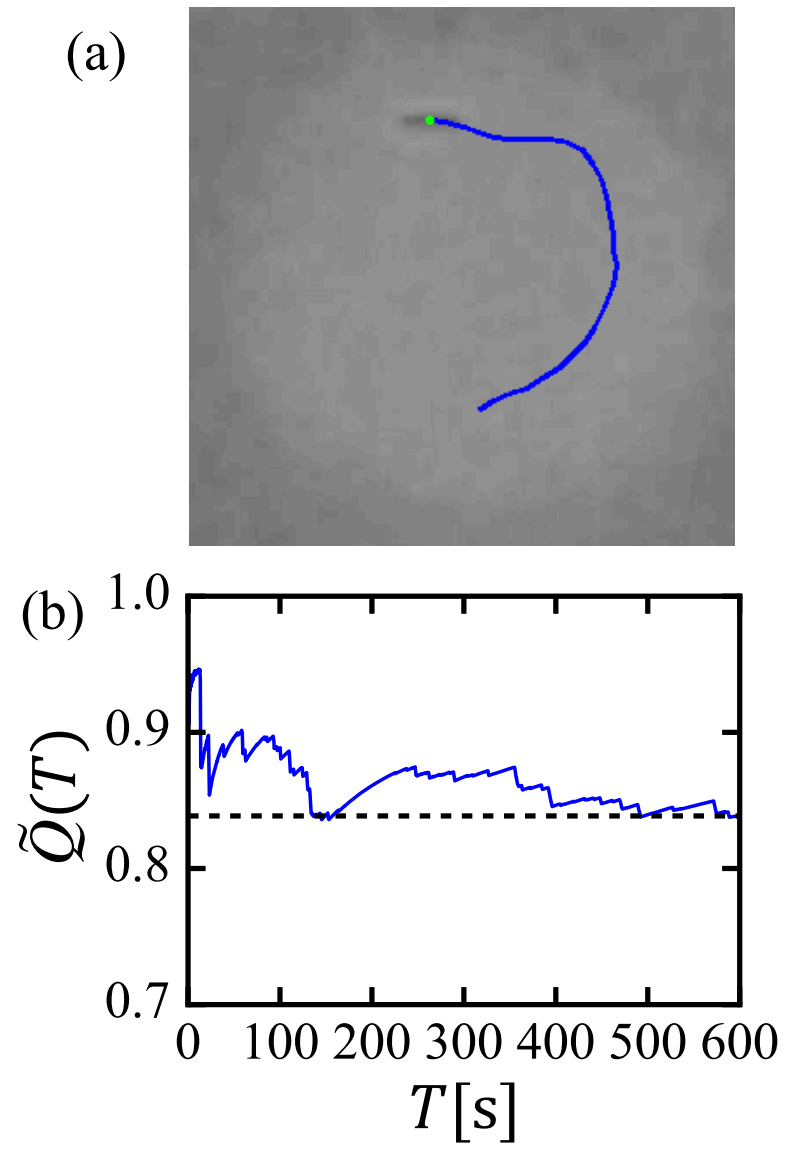

Fig. 4. (Color online) Tracking a bacterial cell in a single cell system. (a) Trajectory of the geometric center of a bacterial cell for $1 \mathrm{~s}$. (b) Time average of the inner products $\tilde{Q}(T)$ defined by Eq. (5) shown as a function of averaging time $T$. 

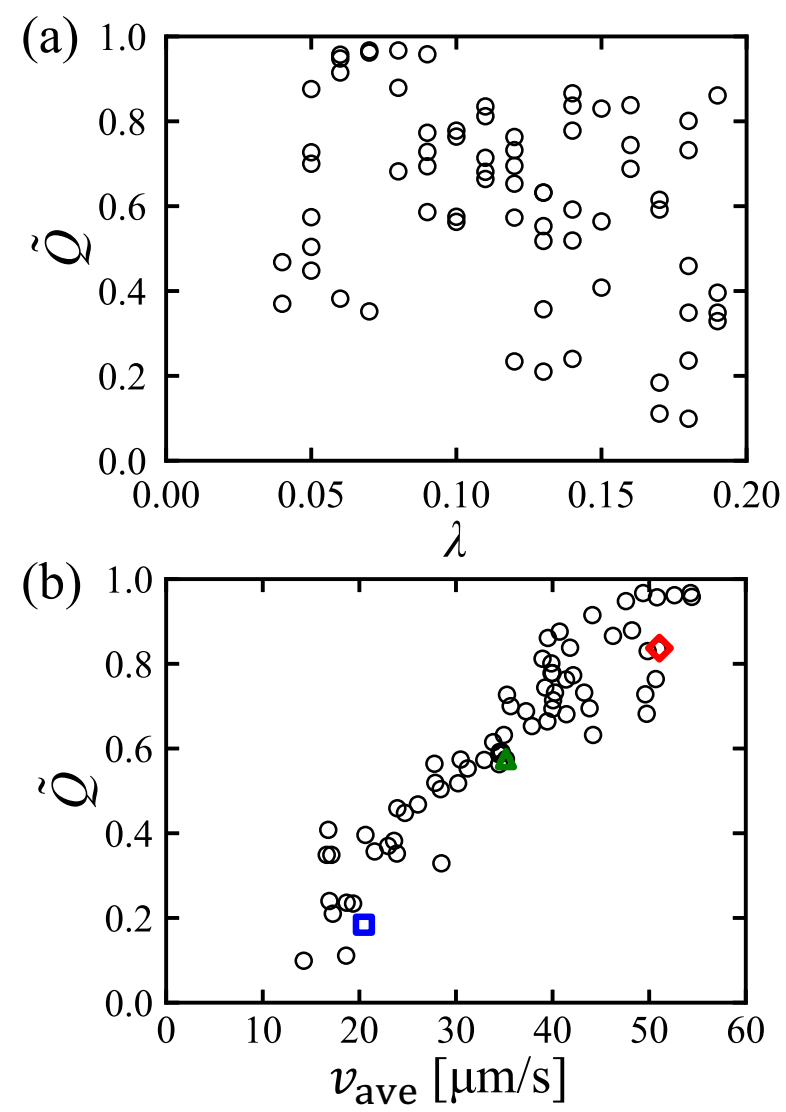

Fig. 5. (Color online) Behavior of the parameter $\tilde{Q}$ for the one-way rotational motion in the single cell systems. (a) Parameter $\tilde{Q}$ plotted against $\lambda(0<\lambda<0.2)$. $\tilde{Q}$ has no systematic dependence on $\lambda$. (b) Parameter $\tilde{Q}$ plotted against $v_{\text {ave }}$. $\tilde{Q}$ seems to increase in proportion to $v_{\text {ave }}$. The coordinates $\left(v_{\text {ave }}[\mu \mathrm{m} / \mathrm{s}], Q\right)$ of the colored symbols shown as $\square$ (blue), $\triangle$ (green), and $\diamond$ (red) are (20.5, 0.18), (35.2, $0.58)$, and $(51.1,0.84)$, respectively. The distributions of cell speed $v$ and the trajectories of a bacterial cell for these plots are shown in Fig. [6. 


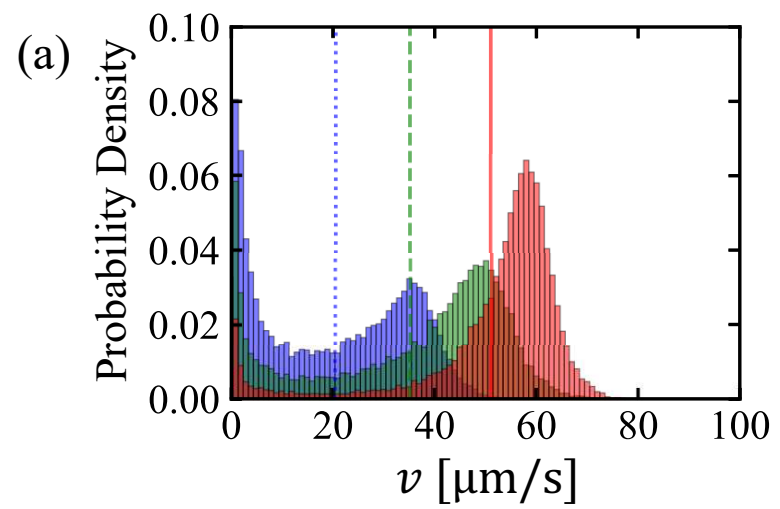

(b)

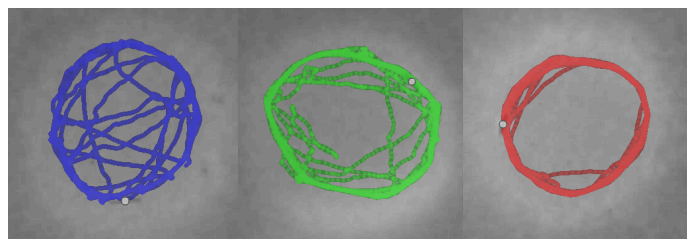

(c)

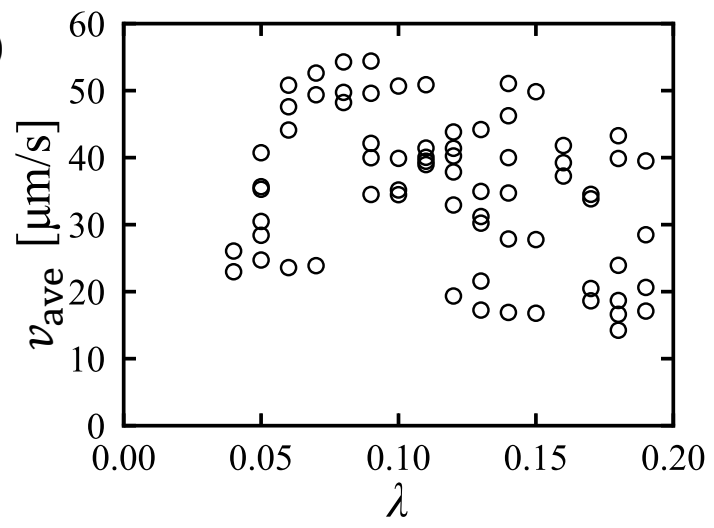

Fig. 6. (Color online) Comparison of the distributions of cell speed $v$. (a) Probability densities of cell speed $v$ distributed in time shown for the different time-average speeds, $v_{\text {ave }}=20.5 \mu \mathrm{m} / \mathrm{s}$ (blue dotted line), $35.2 \mu \mathrm{m} / \mathrm{s}$ (green dashed line), and $51.1 \mathrm{\mu m} / \mathrm{s}$ (red solid line). These values of $v_{\text {ave }}$ correspond to the colored symbols shown as $\square$ (blue), $\triangle$ (green), and $\diamond$ (red) in Fig. 5(b), respectively. Each distribution was made from 18,000 successive speeds $v$. (b) Trajectories of a bacterial cell for the different values of $v_{\text {ave }}$ in (a) shown for $60 \mathrm{~s}$. The left, middle, and right pictures correspond to the distributions of $v$ for $v_{\text {ave }}=20.5,35.2$, and $51.1 \mu \mathrm{m} / \mathrm{s}$, respectively. (c) Values of $v_{\text {ave }}$ plotted against $\lambda$. $v_{\text {ave }}$ did not seem to have any systematic dependence on $\lambda$. 
The behavior of $Q$ reminds us of the dynamical phase transition in the SPP model 1] [16][17] In the SPP model, the transition of the collective motion of self-propelled particles is caused by the competitive relationship between the effect that the individual moving direction is aligned with the averaged moving direction of its neighboring particles and the fluctuations of the moving directions due to noises. There, for a specified particle, neighboring particles are defined as the particles in a circle of some given radius (interaction radius) centered at the position of the specified particle. The transition occurs at the critical noise amplitude or at the critical particle density in the SPP model. On the other hand, in our experiment, the transitions were observed at the critical value of the reduced cell length $\lambda_{\mathrm{C} 1}$. From the microscopic observation, we saw that each rod-shaped bacterial cell had the tendency to be aligned its moving direction with the neighboring cells by the effect of cell-cell interactions. This tendency seemed to be monotonically increasing as the length of bacterial cells increased. Therefore, $\lambda$ is considered to correspond to the interaction radius in the SPP model.

To discuss the effect of cell-cell interactions, we examined the single cell systems by defining the parameter $\tilde{Q}$ for the one-way rotational motion in the single cell systems. Figure 5 (a) shows the plots of the parameter $\tilde{Q}$ against $\lambda$. The values of $\tilde{Q}$ were always positive and were independent of $\lambda$. This is due to the effect of cell-boundary interactions. No critical value $\lambda_{\mathrm{C} 1}$ was found in the plots of $\tilde{Q}$ in the single cell systems having no cellcell interactions. This result indicates that the transitions between the random motion phase and the one-way rotational motion phase are caused by the cell-cell interactions.

In Sect. 3.2.3, we examined the dependence of $\tilde{Q}$ on the time-average speed $v_{\text {ave }} . \tilde{Q}$ seemed to increase in proportion to $v_{\text {ave }}$. When $v_{\text {ave }}$ was sufficiently high, a bacterial cell had the strong tendency to move counterclockwise along the brim of its pool. This yielded a large value of $\tilde{Q}$. When $v_{\text {ave }}$ was low, on the other hand, we obtained a small value of $\tilde{Q}$ as shown in Fig. 5(b). From the distributions of cell speed $v$ for different values of $v_{\text {ave }}$, the distributions were found to have two peaks at $v \cong 0 \mu \mathrm{m} / \mathrm{s}$ and $v>30 \mu \mathrm{m} / \mathrm{s}$ as shown in Fig. 6(a). Under the condition that the total measuring times of $v$ is constant $(18,000$ successive cell speeds in time), when the probability density at $v \cong 0 \mu \mathrm{m} / \mathrm{s}$ was low, that at $v>30 \mu \mathrm{m} / \mathrm{s}$ was high and then $v_{\text {ave }}$ was large. When the probability density at $v \cong 0$ $\mu \mathrm{m} / \mathrm{s}$ was high, that at $v>30 \mu \mathrm{m} / \mathrm{s}$ was low and then $v_{\text {ave }}$ was small. These tendencies suggest that $v_{\text {ave }}$ strongly depends on the relationship between the two peaks. In order to verify this dependence, we simplified the calculation of the time-average speed $v_{\text {ave }}$ by focusing on the two peaks in the distributions. That is, we introduced the simplified average speed $v_{\text {ave }}^{\prime}$ defined by

$$
v_{\text {ave }}^{\prime}=\frac{v_{\mathrm{s}} p_{\mathrm{s}}+v_{\mathrm{l}} p_{\mathrm{l}}}{p_{\mathrm{s}}+p_{\mathrm{l}}}
$$

Here, $v_{\mathrm{s}}$ and $v_{\mathrm{l}}$ are the smaller and larger values of $v$, respectively. $p_{\mathrm{s}}$ is the probability density at $v=v_{\mathrm{s}}$ and $p_{\mathrm{l}}$ is that at $v=v_{\mathrm{l}}$ (the inset in Fig. 8). Figure 8 shows the relationship between $v_{\text {ave }}^{\prime}$ and $v_{\text {ave }}$. $v_{\text {ave }}$ seemed to be linearly dependent on $v_{\text {ave }}^{\prime}$, and to be approximately equal to $v_{\text {ave }}^{\prime}$. Thus, we can conclude that $v_{\text {ave }}$ is almost entirely determined 
by the behavior of the two peaks. In the microscopic observation, a moving bacterial cell was observed to stop to change its direction. Hence, the probability density $p_{\mathrm{s}}$ represents the frequency of the change in the moving direction. In other words, $v_{\text {ave }}$ is considered to have a monotonically decreasing dependence on the frequency of the change in the moving direction. Figure 6(b) shows the trajectories of a bacterial cell for the different values of $v_{\text {ave }}$. There, we see that as $v_{\text {ave }}$ increased, the trajectories became less complicated. When $v_{\text {ave }}=54.3 \mathrm{\mu m} / \mathrm{s}$, the trajectory was almost circular and $\tilde{Q}$ was 0.97 . In this case, the frequency of the change in the moving direction was almost zero and the distribution of $v$ had only one peak at $v \cong 60 \mu \mathrm{m} / \mathrm{s}$ as shown in Fig. 9. The peak at $v=v_{\text {s }}$ disappeared from the distribution of $v$, whereas the peak at $v=v_{1}$ remained in the distribution. Hence, the value of $v_{\mathrm{l}}$ is considered to be the characteristic speed of a bacterial cell in a shallow circular pool. Furthermore, we observed that a bacterial cell sometimes changed its moving direction at the same positions in a pool. Some irregularities may exist at the bottom and the brim of a pool (the surface of an agar plate). Note that the pool had a depth about $1 \mu \mathrm{m}$, which was the same scale as the diameter of a bacterial cell $(\simeq 0.5$ $\mu \mathrm{m})$. Therefore, we attributed the changes in the moving direction of a bacterial cell to the cell-noise interactions, which were regarded as the interactions between each bacterial cell and the irregularities on the surface of a pool. From the above considerations, the behavior of a bacterial cell in the single cell systems was suggested to be dependent on the competition between the cell-boundary interactions and the cell-noise interactions.

From the above considerations, we conclude the following. In this experimental systems, we were able to control the interaction range between bacterial cells by changing $\lambda$. Such a situation is well described in Figs. 3)(b) and 7, which show the $\lambda$-dependence of the order parameter $Q$ defined in Sect. 3.1.1 and its modified version defined in Sect. 4. The scattering of the plots of $Q$ in these graphs is caused by the effect of cell-noise interactions, which were not controlled. Nevertheless, the parameter $\lambda$ is still relevant to describe the transitions, since the critical value $\lambda_{\mathrm{C} 1}$ seemed to be fixed at $\cong 0.1$ so that the order parameter $Q$ evidently increased with $\lambda$ for $\lambda \geq 0.1$ while the dependence of $Q$ on $\lambda$ was negligible for $\lambda<0.1$ for all systems that we examined. Such a sharp transition at $\lambda=\lambda_{\mathrm{C} 1}$ was not confirmed in the single cell systems. Therefore, we conclude that in the transitions between the random motion phase and the one-way rotational motion phase, the cell-cell interactions give the primary effect.

More detailed studies of the cell-noise interactions and of the cell-boundary interactions are required in order to give a more precise description of the transitions including critical phenomena. In the present paper, we have reported our work on the transitions at $\lambda=\lambda_{\mathrm{C} 1}$ with low values of $\rho$. The transitions at $\lambda=\lambda_{\mathrm{C} 1}$ with higher values of $\rho$ (between the turbulent motion phase and the two-way rotational motion phase) as well as those at $\lambda=\lambda_{\mathrm{C} 2}$ will be important future problems. Extensions of the SPP model suitable to describe our experimental results are also required. 


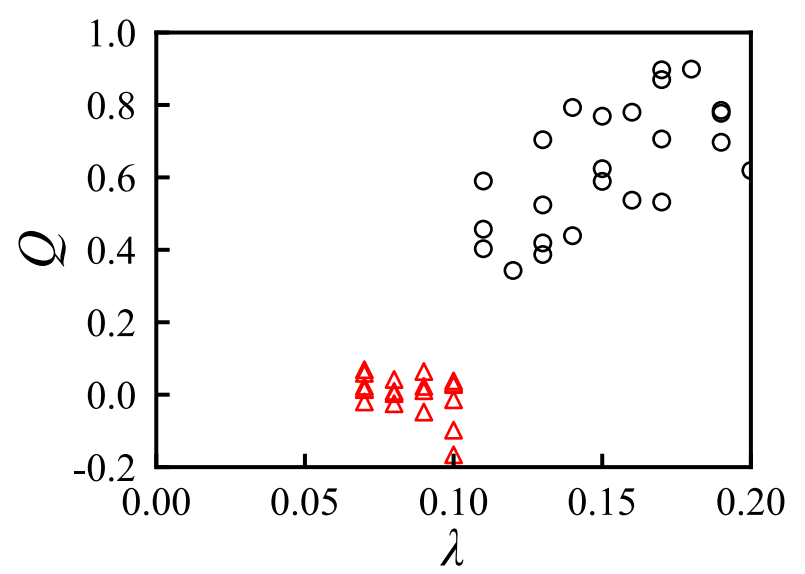

Fig. 7. (Color online) Dependence of the redefined order parameter $Q$ on $\lambda$.

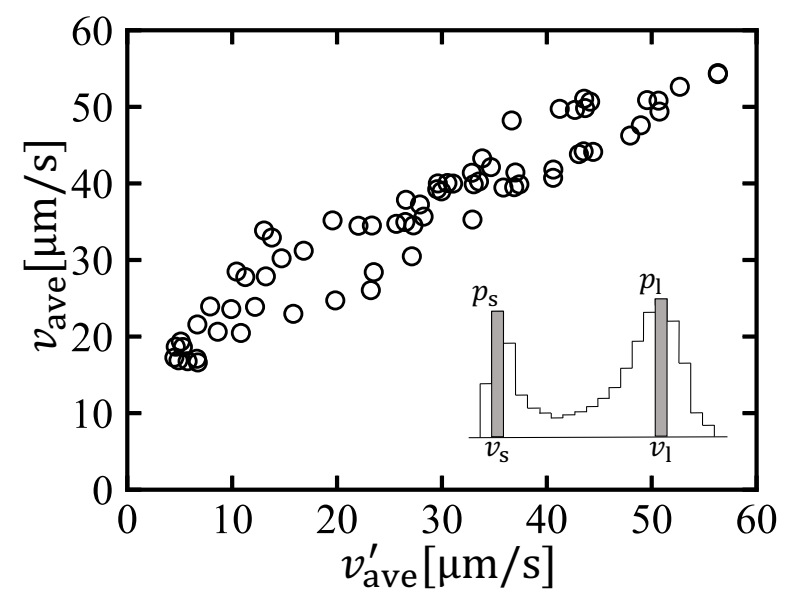

Fig. 8. Relationship between $v_{\text {ave }}^{\prime}$ and $v_{\text {ave }} . v_{\text {ave }}$ seemed to be linearly dependent on $v_{\text {ave }}^{\prime}$. The inset shows a schematic figure of the distribution of cell speeds $v$ in a single cell system. We focused on the two peaks at $v_{\mathrm{s}}(\cong 0 \mu \mathrm{m} / \mathrm{s})$ and $v_{\mathrm{l}}(>30$ $\mu \mathrm{m} / \mathrm{s}) \cdot p_{\mathrm{s}}$ and $p_{\mathrm{l}}$ are the probability densities at $v=v_{\mathrm{s}}$ and $v=v_{\mathrm{l}}$, respectively.

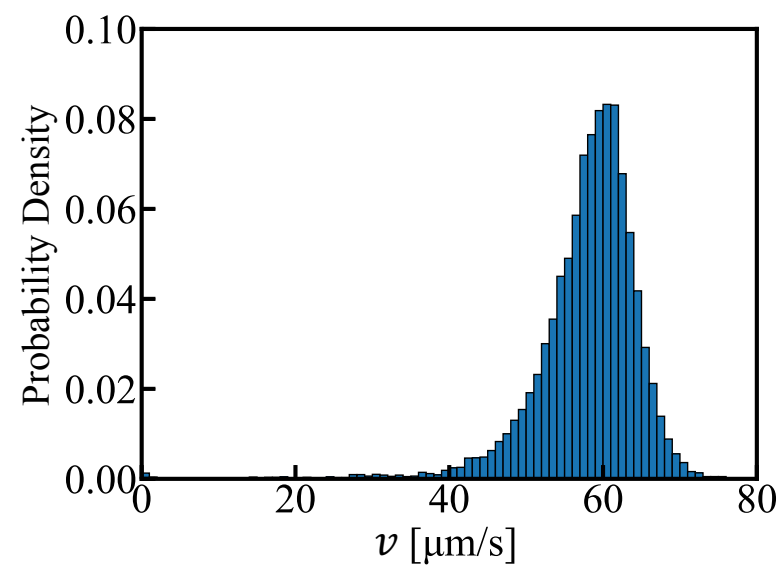

Fig. 9. (Color online) One-peak histogram for the distribution of cell speeds $v$ when $v_{\text {ave }}=54.3 \mu \mathrm{m} / \mathrm{s}$. The histogram was made from 18,000 successive speeds $v$. 


\section{Acknowledgements}

The authors would like to thank Mitsugu Matsushita, Helmut R. Brand, Takuma Narizuka, Yoshihiro Yamazaki, Yusuke T. Maeda, and Hirofumi Wada for useful discussion. They also thank Makoto Katori for careful reading of the manuscript and useful comments. JW is supported by a Chuo University Grant for Special Research and by a Grant-in-Aid for Exploratory Research (No. 15K13537) from the Japan Society for the Promotion of Science (JSPS).

1) T. Vicsek and A. Zafeiris, Phys. Rep. 517, 71 (2012).

2) M. Ballerini, N. Cabibbo, R. Candelier, A. Cavagna, E. Cisbani, I. Giardina, V. Lecomte, A. Orlandi, G. Parisi, A. Procaccini, M. Viale, and V. Zdravkovic, Proc. Natl. Acad. Sci. U.S.A. 105, 1232 (2008).

3) A. Cavagna, A. Cimarelli, I. Giardina, G. Parisi, R. Santagati, F. Stefanini, and M. Viale, Proc. Natl. Acad. Sci. U.S.A. 107, 11865 (2010).

4) C. K. Hemelrijk, H. Hildenbrandt, J. Reinders, and E. J. Stamhuis, Ethology 116, 1099 (2010).

5) C. Becco, N. Vandewalle, J. Delcourt, and P. Poncin, Physica A 367, 487 (2006).

6) A. Czirók, E. Ben-Jacob, I. Cohen, and T. Vicsek, Phys. Rev. Lett. 54, 1791 (1996).

7) J. Wakita, I. Ràfols, H. Itoh, T. Matsuyama, and M. Matsushita, J. Phys. Soc. Jpn. 67, 3630 (1998).

8) A. Sokolov and I. S. Aranson, Phys. Rev. Lett. 109, 248109 (2012).

9) H. H. Wensink, J. Dunkel, S. Heidenreich, K. Drescher, R. E. Goldstein, H. Löwen, and J. M. Yeomans, Proc. Natl. Acad. Sci. U.S.A. 109, 14308 (2012).

10) R. Honda, J. Wakita, and M. Katori, J. Phys. Soc. Jpn. 84, 114002 (2015).

11) G. Mamou, G. B. M. Mohan, A. Rouvinski, A. Rosenberg, and S. Ben-Yehuda, Cell Rep. 14, 1850 (2016).

12) M. Matsushita, F. Hiramatsu, N. Kobayashi, T. Ozawa, Y. Yamazaki, and T. Matsuyama, Biofilms 1, 305 (2004).

13) A. Nakahara, Y. Shimada, J. Wakita, M. Matsushita, and T. Matsuyama, J. Phys. Soc. Jpn. 65, 2700 (1996).

14) F. Hiramatsu, J. Wakita, N. Kobayashi, Y. Yamazaki, M. Matsushita, and T. Matsuyama, Microbes Environ. 20, 120 (2005). 
15) R. Tokita, T. Katoh, Y. Maeda, J. Wakita, M. Sano, T. Matsuyama, and M. Matsushita, J. Phys. Soc. Jpn 78, 074005 (2009).

16) T. Vicsek, Fluctuations and Scaling in Biology (Oxford University Press, New York, 2001).

17) T. Vicsek, A. Czirók, E. Ben-Jacob, I. Cohen, and O. Shochet, Phys. Rev. Lett. 75, 1226 (1995).

18) A. Czirók, H. E. Stanley, and T. Vicsek, J. Phys. A 30, 1375 (1997).

19) H. Chaté, F. Ginelli, G. Grégoire, and F. Raynaud, Phys. Rev. E 77, 046113 (2008).

20) G. Baglietto, E. V. Albano, and J. Candia, Interface Focus 2, 708 (2012).

21) H. Wioland, F. G. Woodhouse, J. Dunkel, J. O. Kessler, and R. E. Goldstein, Phys. Rev. Lett. 110, 268102 (2013).

22) H. Wioland, F. G. Woodhouse, J. Dunkel, and R. E. Goldstein, Nat. Phys. 12, 341 (2016).

23) K. Beppu, Z. Izri, J. Gohya, K. Eto, M. Ichikawa, and Y. T. Maeda, Soft Matter 13, 5038 (2017).

24) D. Nishiguchi, I. S. Aranson, A. Snezhko, and A. Sokolov, Nat. Commun. 9, 4486 (2018).

25) K. T. Wu and J. B. Hishamunda, Science 355, 6331 (2017).

26) A. Bricard, J.-B. Caussin, D. Das, C. Savoie, V. Chikkadi, K. Shitara, O. Chepizhko, F. Peruani, D. Saintillan, and D. Bartolo, Nat. Commun. 6, 7470 (2015).

27) A. Deblais, T. Barois, T. Guerin, P. H. Delville, R. Vaudaine, J. S. Lintuvuori, J. F. Boudet, J. C. Baret, and H. Kellay Phys. Rev. Lett. 120, 188002 (2018).

28) J. Wakita, S. Tsukamoto, K. Yamamoto, M. Katori, and Y. Yamada, J. Phys. Soc. Jpn. 84, 124001 (2015).

29) J. Wakita, H. Shimada, H. Itoh, T. Matsuyama, and M. Matsushita, J. Phys. Soc. Jpn. 70, 911 (2001). 